\title{
NNLL Electroweak Corrections to Gauge Boson Pair Production at LHC
}

\author{
Sandro Uccirati* \\ Institut für Theoretische Teilchenphysik, Universität Karlsruhe, \\ 76128 Karlsruhe, Germany \\ E-mail: ucciratieparticle.uni-karlsruhe.de
}

The high energy behaviour of electroweak corrections to gauge boson pair production at LHC can be derived in the framework of evolution equations up to NNLL contributions to all order in perturbation theory. On the basis of this general result we derive the one- and two-loop cross section in NNLL approximation for W-pair production at partonic and hadronic level.

European Physical Society Europhysics Conference on High Energy Physics, EPS-HEP 2009, July 16 - 222009

Krakow, Poland

\footnotetext{
* Speaker.

${ }^{\dagger}$ In collaboration with J.H.Kühn, F.Metzler, A.A.Penin
} 


\section{Introduction}

The most important goal of the LHC is the search for the Higgs Boson and one of its discovery channels is the reaction $p p \rightarrow H \rightarrow W^{+} W^{-}$. The production of a pair of $W$ bosons constitutes therefore an important background for Higgs production and decay at LHC. In addition, the comparison of precise measurements of the production of gauge boson pairs with theoretical predictions is a good test of the gauge trilinear couplings predicted by the Standard Model. The LHC will exploit the high energy region where the $\mathrm{W}$ bosons can be considered as light and the main contribution to the cross section comes from large Sudakow logarithms [1].

\section{Evolution equations for $S U(N)$ and for the electroweak SM}

In a $S U(N)$ gauge theory in the high energy limit, where all the kinematical invariants are of the same order and far larger than the gauge boson mass, $|s| \sim|t| \sim|u| \gg M^{2}$, the asymptotic energy dependence of the amplitude is dominated by Sudakov logarithms $\log \left(Q^{2} / M^{2}\right)\left(Q^{2}=-s\right)$ and easily studied within the evolution equations framework.

The logarithms of collinear and soft-collinear origin depend only on the properties of the external particles and are known to factorize. They do not depend on the specific process and can be determined studying the asymptotic behaviour of the scattering form factors depicted in Fig. 1.
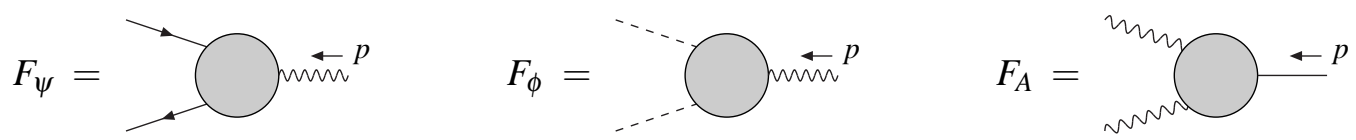

Figure 1: Fermion/scalar scattering in an external singlet vector field and scattering of a gauge boson in an external scalar field. The momentum of external field satisfies $p^{2}=s=-Q^{2}$.

At leading order in $M^{2} / Q^{2}$ they are known to satisfy the following evolution equation [2]

$$
\begin{aligned}
& F_{i}=Z_{i} F_{i}^{\text {Born }}, \quad \frac{\partial Z_{i}}{\partial \ln Q^{2}}=\left[\int_{M^{2}}^{Q^{2}} \frac{d x}{x} \gamma_{i}\left(g^{2}(x)\right)+\zeta_{i}\left(g^{2}\left(Q^{2}\right)\right)+\xi_{i}\left(g^{2}\left(M^{2}\right)\right)\right] Z_{i}, \quad i=\psi, \phi, A \\
& Z_{i}=Z_{i, 0}\left(g^{2}\left(M^{2}\right)\right) \exp \left\{\int_{M^{2}}^{Q^{2}} \frac{d x}{x}\left[\int_{M^{2}}^{x} \frac{d x^{\prime}}{x^{\prime}} \gamma_{i}\left(g^{2}\left(x^{\prime}\right)\right)+\zeta_{i}\left(g^{2}(x)\right)+\xi_{i}\left(g^{2}\left(M^{2}\right)\right)\right]\right\} .
\end{aligned}
$$

The remaining soft logarithms (not contained in $Z_{i}$ ) depend on the process under consideration. We concentrate now on $2 \rightarrow 2$ processes, where the incoming particles transform under the fundamental representation of the group (as happens for processes with incoming fermions). If $T_{a}$ are the generators of the algebra, for final states in the fundamental and adjoint representation the structure of the amplitude is $A_{F}=A_{1} T_{a} \times T_{a}+A_{2} \mathbb{1} \times \mathbb{1}$ and $A_{A}=A_{1} T_{a} T_{b}+A_{2} T_{b} T_{a}+A_{3} \delta_{a b} \mathbb{1}$ respectively. The coefficients $A_{j}$ can be seen as the components of a vector $\vec{A}$. Factorizing out of $\vec{A}$ the $Z_{i}$ factors described above, the remaining reduced amplitude satisfies a renormalization group like equation [3] whose solution is given by a path-ordered exponent:

$$
\vec{A}=\prod_{n=1}^{4} Z_{i_{n}}^{1 / 2} \vec{A}^{\prime}, \quad \frac{\partial \vec{A}^{\prime}}{\partial \ln Q^{2}}=\chi\left(g^{2}\left(Q^{2}\right)\right) \vec{A}^{\prime}, \quad \vec{A}^{\prime}=\mathrm{P} \exp \left[\int_{M^{2}}^{Q^{2}} \frac{\mathrm{d} x}{x} \chi\left(g^{2}(x)\right)\right] \vec{A}_{0}\left(g^{2}\left(M^{2}\right)\right)
$$


where $i_{n}$ takes the values $\psi, \phi$ or $A$ depending on whether the $n^{\text {th }}$ external particle is a fermion, a scalar or a vector boson and $\chi$ is the soft anomalous dimension matrix. Expanding all perturbative functions of Eq.(2.1) and Eq.(2.2) in $g^{2}\left(\mu^{2}\right)$ and absorbing $Z_{i, 0}$ in $\vec{A}_{0}$, we get:

$$
\begin{aligned}
& \vec{A}^{(1)}=\frac{1}{2} \gamma^{(1)} \vec{A}^{\text {Born }} L^{2}+\left(\zeta^{(1)}+\xi^{(1)}+\chi^{(1)}\right) \vec{A}^{\text {Born }} L+\vec{A}_{0}^{(1)}, \quad L=\log \frac{Q^{2}}{M^{2}} \\
& \vec{A}^{(2)}=\frac{1}{8}\left[\gamma^{(1)}\right]^{2} \vec{A}^{\text {Born }} L^{4}+\frac{\gamma^{(1)}}{2}\left[\zeta^{(1)}+\xi^{(1)}+\chi^{(1)}-\frac{\beta_{0}}{3}\right] \vec{A}^{\text {Born }} L^{3} \\
& +\frac{1}{2}\left\{\left[\gamma^{(2)}+\left(\zeta^{(1)}+\xi^{(1)}\right)^{2}-\beta_{0} \zeta^{(1)}+\left(\chi^{(1)}\right)^{2}-\beta_{0} \chi^{(1)}\right] \vec{A}^{\text {Born }}+\vec{A}_{0}^{(1)}\right\} L^{2}+\mathscr{O}(L)
\end{aligned}
$$

where $\{\gamma, \zeta, \xi\}=1 / 2 \sum_{n=1}^{4}\left\{\gamma_{i_{n}}, \zeta_{i_{n}}, \xi_{i_{n}}\right\}$. For the running of the coupling constant we have used $g^{2}(x)=g^{2}\left(M^{2}\right)\left[1-g^{2}\left(M^{2}\right) \beta_{0} \log \left(x / M^{2}\right)\right]$. The one-loop coefficients can be fixed by comparing the first expression in Eq.(2.3) with the asymptotic one-loop result (see [4] for explicit values). They are sufficient to determine throught the second equation in Eq.(2.3) the leading and next-toleading logarithms at two-loop level, while to compute the two-loop NNLL one needs in addition $\gamma_{i}^{(2)}$, which is also known ([5]).

In the electroweak Standard Model, which is a $S U(2)_{L} \times U(1)_{Y}$ theory, the situation is complicated by the mixing of the two gauge groups as a consequence of the symmetry breaking and by the presence of two irreducible scales: the electroweak scale $M \sim M_{W} \sim M_{Z} \sim M_{H}$ and the infrared scale $\lambda$. In order to proceed without breaking gauge invariance [6], one considers in a first step the case where the SM obeys the evolution equations of an unbroken $S U(2)_{L} \times U(1)_{Y}$ theory and all gauge bosons have the same mass $(\lambda=M)$. The amplitude takes the simple form $U_{Y}(M) \vec{A}_{L}(M)$ where $\vec{A}_{L}$ is obtained from Eq.(2.3) for the group $S U(2)_{L}$ (with $g^{2} \rightarrow \alpha /\left(4 \pi s_{W}^{2}\right)$ ) and $U_{Y}$ is the hypercharge $U(1)$ factor. All logarithms are here of the form $\ln \left(Q^{2} / M^{2}\right)$. Then we move to the (physical) case where $\lambda \ll M$ and the infrared logarithms $\ln \left(Q^{2} / \lambda^{2}\right)$ are described by the $U(1)$ QED factor $U_{Q E D}$ up to power suppressed terms in $\lambda / M$. Requiring the matching of the two regions at $M=\lambda$ leads to the result:

$$
\vec{A}(M, \lambda)=\left.C(M) U_{Q E D} U_{Q E D}^{-1}\right|_{\lambda=M} U_{Y} \vec{A}_{L} .
$$

The matching coefficient $C(M)$ describes the effect of the neglected terms in $\lambda / M$. This strategy has been used at NNLL level in many similar studies [4].

The chiral structure of the SM can be taken into account enlarging the basis in which the vector $\vec{A}$ is defined, while the mass splitting between the bosons at the electroweak scale can be efficiently included in the parameter $\xi$ [4]. The effect of the top Yukawa coupling are also considered following the approach described in the last reference of [4].

\section{W-pair production at hadron colliders}

The partonic processes relevant for the W-pair production at hadron colliders are the gluon fusion and quark-antiquark annihilation. The contribution of the former to the total cross section is about $5 \%$, therefore we concentrate our analysis on the process $q \bar{q} \rightarrow W^{+} W^{-}$which is described at leading order by the first two diagrams depicted in Fig. 2. In the high energy limit only final states where both $W$ 's have the same polarization are not mass suppressed. In addition, the case where 
both $W$ 's are longitundinal can be reduced by means of the Goldstone theorem to the production of a pair of charged Goldstone bosons, reducing the study of the process to the last two diagrams of Fig. 2. We have explicitly computed the one-loop corrections to these diagrams and fixed the
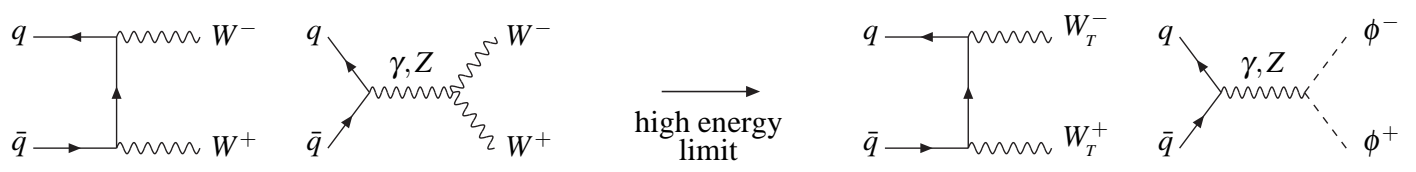

Figure 2: Tree level diagrams contributing to the partonic process

one-loop coefficients of the evolution equations by which we have determined the two-loop leading logarithms (up to NNLL). In Fig. 3 the one- and two-loop corrections for left-handed $u$ quarks in the initial state are plotted showing an important contribution at NNLL which partially cancels the NLL. The behaviour for the $d$ quarks is similar, while the right-handed contribution is negligible.
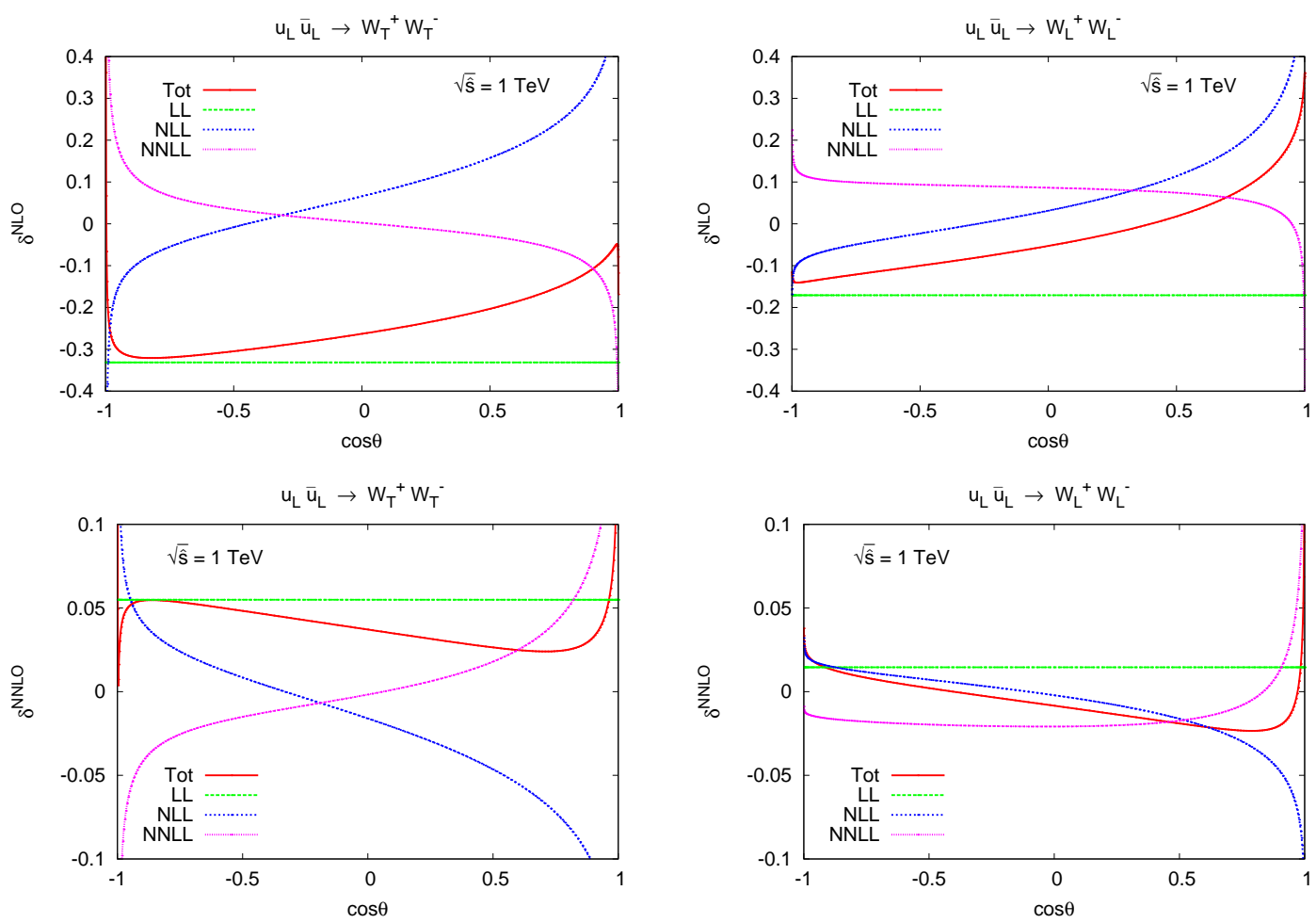

Figure 3: One- and two-loop corrections to the partonic cross section for left-handed $u$ quarks with energy $\sqrt{\hat{s}}=1 \mathrm{TeV}\left(\delta^{i}=d \sigma_{i} / d \sigma_{\mathrm{LO}}, i=N L O, N N L O\right)$.

The hadronic distributions are then obtained folding the partonic cross section with the parton distribution functions. For the $p_{T}$-distribution we have:

$$
\begin{gathered}
\frac{d \sigma}{d p_{T}}=\frac{1}{N_{c}^{2}} \sum_{i j} \int_{0}^{1} d x_{1} \int_{0}^{1} d x_{2} f_{h_{1}, i}\left(x_{1}, \mu_{F}^{2}\right) f_{h_{2}, j}\left(x_{2}, \mu_{F}^{2}\right) \theta\left(x_{1} x_{2}-\tau_{\min }\right) \frac{d \hat{\sigma}_{i j}}{d p_{T}} \\
\frac{d \hat{\sigma}_{i j}}{d p_{T}}=\frac{4 p_{T}}{\sqrt{\hat{s}-4 M_{W}^{2}} \sqrt{\hat{s}-s \tau_{\min }}}\left[\frac{d \hat{\sigma}_{i j}}{d \cos \theta}+(\hat{t} \leftrightarrow \hat{u})\right] \quad \hat{s}=x_{1} x_{2} s \quad \tau_{\min }=\frac{4\left(p_{T}^{2}+M_{W}^{2}\right)}{s}
\end{gathered}
$$


Restricting the analysis to high $p_{T}$ values, the partonic process is in the range of validity of the high energy limit. The results, plotted in Fig. 4, show that the NNLO corrections at high energies are between $1 \%$ and $25 \%$ for transversal $W$ 's and between $-1 \%$ and $3 \%$ in the longitudinal case.
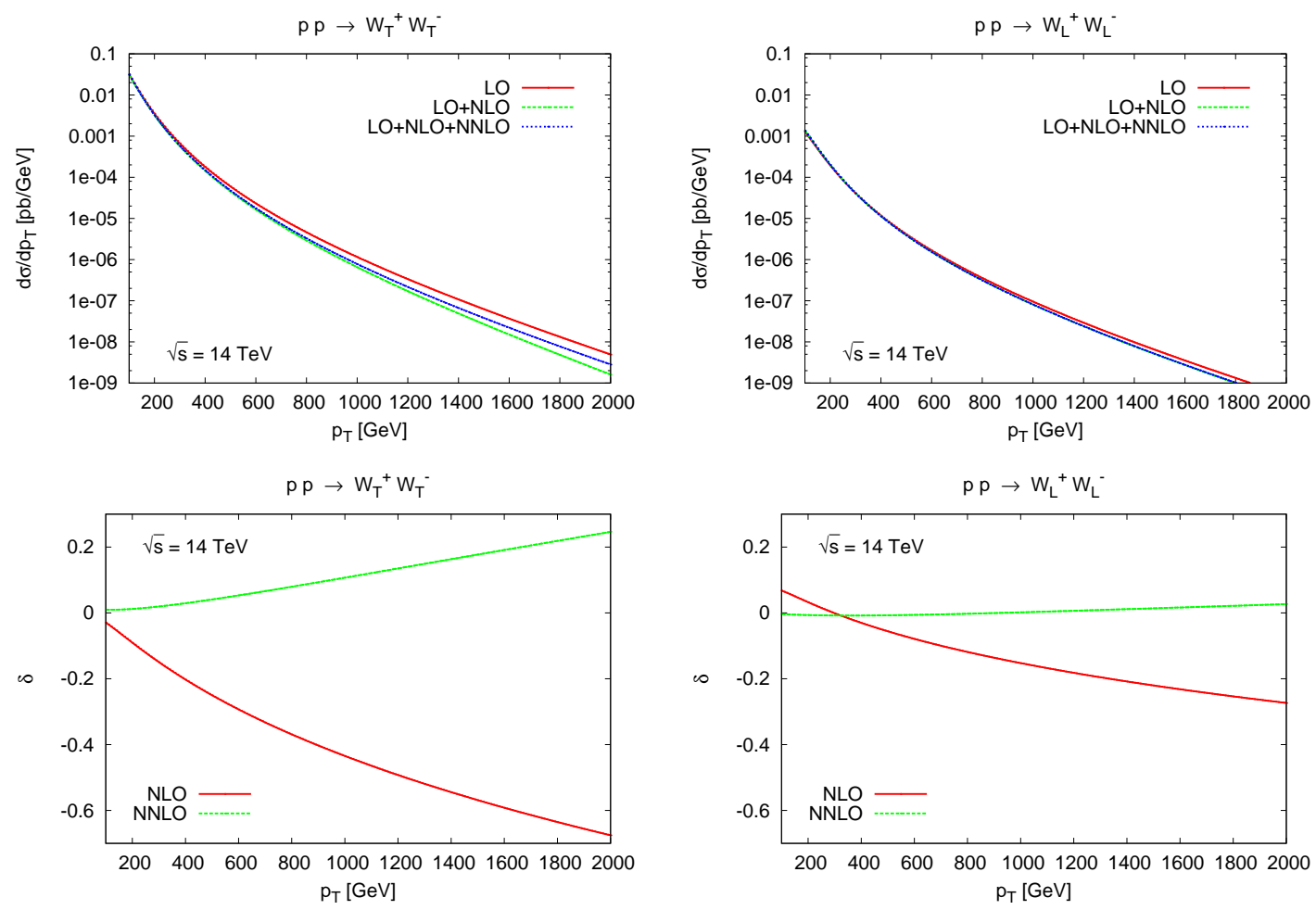

Figure 4: Hadronic $p_{T}$ distributions at LHC for beem energy of $14 \mathrm{TeV}\left(\delta^{i}=d \sigma_{i} / d \sigma_{\mathrm{LO}}, i=N L O, N N L O\right)$.

\section{References}

[1] V. V. Sudakov, Sov. Phys. JETP 3 (1956) 65 [Zh. Eksp. Teor. Fiz. 30 (1956) 87].

[2] A. H. Mueller, Phys. Rev. D 20 (1979) 2037. J. C. Collins, Phys. Rev. D 22 (1980) 1478, Adv. Ser. Direct. High Energy Phys. 5 (1989) 573 A. Sen, Phys. Rev. D 24 (1981) 3281.

[3] A. Sen, Phys. Rev. D 28 (1983) 860. G. Sterman, Nucl. Phys. B 281 (1987) 310. J. Botts and G. Sterman, Nucl. Phys. B 325 (1989) 62.

[4] J. H. Kuhn, A. A. Penin and V. A. Smirnov, Eur. Phys. J. C 17 (2000) 97 J. H. Kuhn, S. Moch, A. A. Penin and V. A. Smirnov, Nucl. Phys. B 616 (2001) 286 [Erratum-ibid. B 648 (2003) 455]

B. Feucht, J. H. Kuhn, A. A. Penin and V. A. Smirnov, Phys. Rev. Lett. 93 (2004) 101802 B. Jantzen, J. H. Kuhn, A. A. Penin and V. A. Smirnov, Nucl. Phys. B 731 (2005) 188 [Erratum-ibid. B 752 (2006) 327] J. H. Kuhn, F. Metzler and A. A. Penin, Nucl. Phys. B 795 (2008) 277 [Erratum-ibid. 818 (2009) 135]

[5] J. Kodaira and L. Trentadue, Phys. Lett. B 112 (1982) 66. C. T. H. Davies and W. J. Stirling, Nucl. Phys. B 244 (1984) 337. G. P. Korchemsky and A. V. Radyushkin, Nucl. Phys. B 283 (1987) 342.

[6] V. S. Fadin, L. N. Lipatov, A. D. Martin and M. Melles, Phys. Rev. D 61 (2000) 094002 M. Melles, Phys. Rev. D 63 (2001) 034003 